\title{
Finding the upper mass limit until which gravimetric sensors preserve their original TCF and sensitivity
}

\author{
Teona Mirea, Marta Clement, Jimena Olivares, Enrique Iborra
}

\begin{abstract}
Thin film bulk acoustic wave resonators (FBARs) have undoubtedly overcome the mass sensitivity of their predecessors quartz crystal microbalances. However, the mass sensitivity of FBARs strongly depends on all layers composing their complex structure. It has been proved that the addition of materials with different acoustic impedances at the sensing surface of the device can vary their sensitivity by energy redistribution effects. Such materials have been also used for temperature coefficient (TCF) compensation. Here we aim at studying up to which thickness of an added specific material on AIN-based solidly mounted resonators (SMRs), the initial mass sensitivity and TCF are still preserved. We prove that the sensitivity can be considered lineal up to the deposition of around $100 \mathrm{~nm}$ of $\mathrm{SiO}_{2}$, not affecting the detection of small masses in the pg range. On the contrary, TCF variation with few $\mathrm{nm}$ of $\mathrm{SiO}_{2}$ needs to be accurately controlled since it affects the detection in the pg range. For the detection of heavy masses, these effects can be considered negligible. This study should be performed for each particular case depending on the material accumulated on the device.
\end{abstract}

Keywords-AlN-based SMR; mass sensitivity; temperature coefficient of frequency.

\section{INTRODUCTION}

Thin film acoustic wave resonators working in the $\mathrm{GHz}$ range of frequency, particularly the ones based on bulk waves (FBARs), have overcome the mass sensitivity of their quartz crystal microbalance predecessors [1]. However, in contrast to the QCM sensitivity, which only depends on the thick quartz plate characteristics and the ones of the sensed (or deposited) material [2], the sensitivity of FBARs depends on the characteristics of all the films composing the multilayered structure including the one formed by the sensed material [3]. To accurately use FBARs as gravimetric sensors, either in liquid or gaseous media, factors such as the temperature coefficient of frequency (TCF) that accounts for the influence of the ambient temperature on the sensor frequency shifts, needs to be considered. If the TCF value is well-known, it can be used for measurements correction. Numerous studies on the TCF analysis of FBARs have been carried out, most particularly on their zero TCF compensation, which can be achieved by adding a $\mathrm{SiO}_{2}$ film to the device structure [4]-[6]. Most of them investigate the appropriate $\mathrm{SiO}_{2}$ thickness to get the full compensation, however none of them discuss the maximum $\mathrm{SiO}_{2}$ (or other material) thickness up to which the initial TCF does not vary. Another important issue is the variation of the device sensitivity due to the energy trapping effect, i.e. the sensitivity variation with the increased deposited material [3]. Both effects need to be considered in biosensors with complex functionalization or in mass sensors that do not recover the initial state (mass accumulation).

In this work we adopt a reverse view, compared to the existing studies, and we aim at finding the thickness limit of a given film deposited on solidly mounted resonators (SMRs), up to which the initial TCF and sensitivity values of the device are still preserved in a given range. We study both the shear and longitudinal modes, which are typically used for biosensing (liquid) and gas phase sensing applications, respectively. We used both theoretical predictions based on Mason's model, and experimental results. Based on the obtained results we will discuss if SMR gravimetric sensors can be reused or not in different mass sensing applications.

\section{A. Devices}

We use SMRs based on fully-insulating acoustic reflectors made of seven alternating layers of $\mathrm{SiO}_{2} / \mathrm{AlN}$, on which we deposit a tilted piezoelectric AlN film sandwiched between two electrodes, Ir and Mo as bottom and top electrodes, respectively (Fig. 1a). The acoustic reflector was designed to properly confine (reflect) the acoustic energy of the shear mode operating at around $1.5 \mathrm{GHz}$ and the longitudinal mode at around $2.5 \mathrm{GHz}$ (Fig. 1b).

\section{B. Characterization}

As testing film deposited on the surface of our SMRs we used $\mathrm{SiO}_{2}$ sputtered sequentially up to $300 \mathrm{~nm}$, which corresponds to $66 \mu \mathrm{g} / \mathrm{cm}^{2}\left(0.22 \mu \mathrm{g} / \mathrm{mm} \cdot \mathrm{cm}^{2}\right.$ considering a density of $\left.2.2 \mathrm{~g} / \mathrm{cm}^{3}\right)$. The mass sensitivity $\left(S_{m}\right)$ and TCF values were tested after each deposition, allowing us to determine if the previous deposited film had an influence on the new structure characteristics. Their values were calculated according to:

$$
\begin{aligned}
& S_{m}=(\Delta f) /\left(\Delta \mathrm{t} \cdot \rho_{\mathrm{SiO} 2}\right) \\
& \mathrm{TCF}=\left(\Delta f l f_{0}\right) /(\Delta \mathrm{T})
\end{aligned}
$$

where $\Delta \mathrm{t}$ and $\rho_{\mathrm{SiO} 2}$ stand for the deposited thickness and density of the $\mathrm{SiO}_{2}$ layer (i.e. the mass of $\Delta \mathrm{t}$ per unit of area), and $\Delta f$ 
(a)

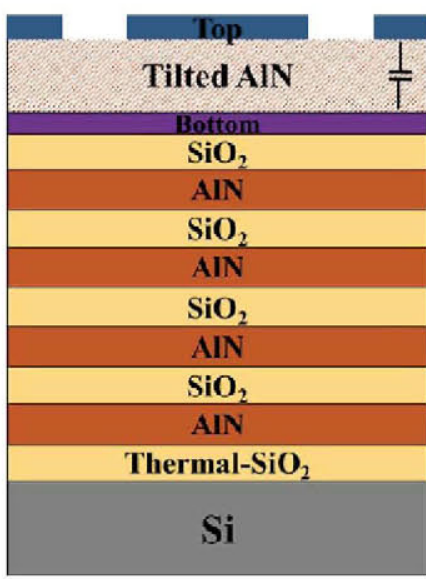

(b)

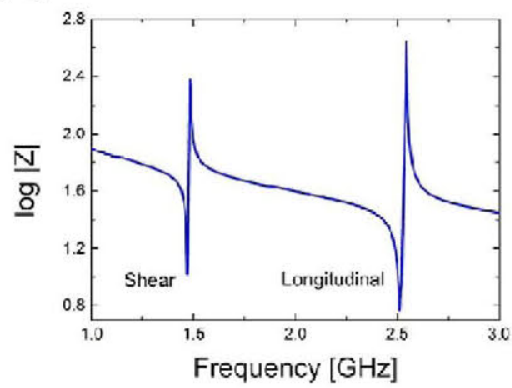

Fig. 1. Cross sectional sketch of the AIN-based SMR used (a) and its electrical impedance response showing the presence of both shear and longitudinal modes.

and $\Delta \mathrm{T}$ for the frequency and temperature shifts; $f_{0}$ is the starting frequency.

For the TCF assessment we subjected the devices to temperatures ranging from $20^{\circ} \mathrm{C}$ to $100^{\circ} \mathrm{C}$ using a Peltier cell (Fig. 2) and monitored the device response by tracking the frequency of the admittance real part $(\operatorname{Re}(\mathrm{Y}))$ maximum through a specifically designed LabView( $\left.{ }^{\circledR}\right)$ application. This procedure is based on acquiring the $\operatorname{Re}(\mathrm{Y})$ maximum around the resonant frequency with a bandwidth of $20 \mathrm{MHz}$ and fitting it to a rational function. Floor-noise levels under $500 \mathrm{~Hz}$ as a function of time were systematically achieved.

\section{RESULTS AND DISCUSSION}

Fig. 3 shows the simulated and experimental results of both the longitudinal and shear sensitivities against the previously deposited $\mathrm{SiO}_{2}$ thickness. That is, $x$ axis values are the thickness of the $\mathrm{SiO}_{2}$ film that the device had on the top electrode prior to a new sensing event, while $\Delta x$ values between each point correspond to the sensed thickness, i.e. $\Delta t$ in Eq. 2. As expected, we observe that the mass sensitivity of the longitudinal mode is higher than for the shear mode, and while the experimental values for the shear mode fit pretty well the simulated results, the experimental measurements for the longitudinal mode have a considerable deviation at lower thicknesses of $\mathrm{SiO}_{2}$. We attribute this to a possible difference in
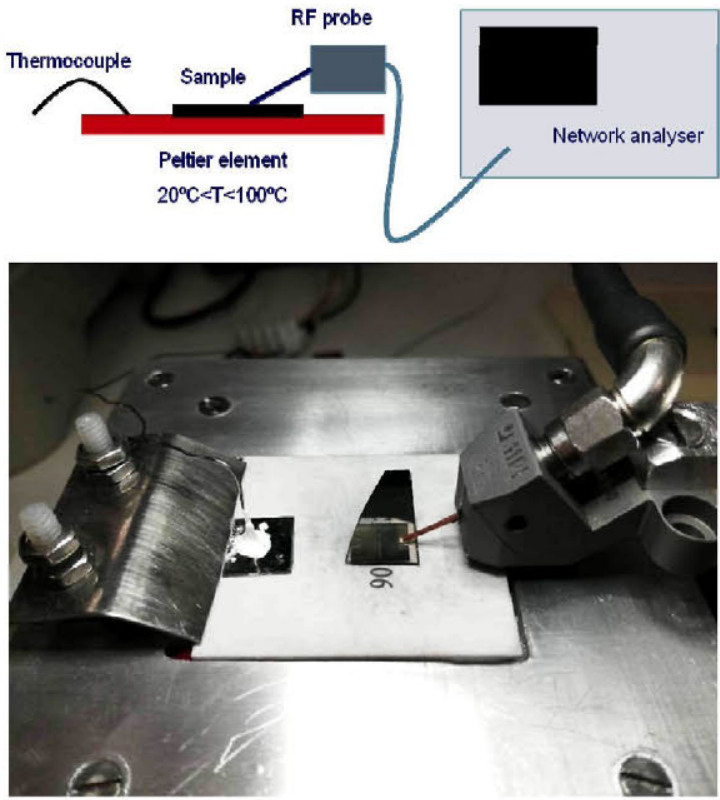

Fig. 2. Measurment set-up for the TCF characterization.

the longitudinal acoustic characteristic of real and simulated $\mathrm{SiO}_{2}$ films. It is evident that the mass sensitivity starts deviating from its initial value (i.e. without $\mathrm{SiO}_{2}$ ) with very few $\mathrm{nm}$ of deposited $\mathrm{SiO}_{2}$. It is reduced up to around $100 \mathrm{~nm}$, value after which starts having a considerable increase (curves decay). For the shear mode, up to approximately $100 \mathrm{~nm}$ of deposited $\mathrm{SiO}_{2}$, the sensitivity moves between $-2.55 \cdot 10^{12} \mathrm{~Hz} \cdot \mathrm{cm}^{2} / \mathrm{g}$ and $-2.7 \cdot 10^{12} \mathrm{~Hz} \cdot \mathrm{cm}^{2} / \mathrm{g}$, which means an average deviation of around $-0.15 \cdot 10^{12} \mathrm{~Hz} \cdot \mathrm{cm}^{2} / \mathrm{g}$. Considering that our target would be the detection of $\Delta \mathrm{m}=1 \mathrm{ng} / \mathrm{cm}^{2}$, that would imply a shift in frequency of around 2.6. In this case the error in detection $\Delta f_{2}-$ $\Delta f_{1}=0.15 \mathrm{kHz}$ is much lower than our resolution, hence allowing us to consider a constant sensitivity of $S_{m}=-2.7 \cdot 10^{12}$ $\mathrm{Hz} \cdot \mathrm{cm}^{2} / \mathrm{g}$ for devices with up to $100 \mathrm{~nm}$ of deposited $\mathrm{SiO}_{2}$. Of course, $\mathrm{SiO}_{2}$ is just a particular case here and the study should be extended for each material case. The same conclusion could be applied for the longitudinal case, where the sensitivity seems to keep a relatively small variation (according to the simulation) compared to its pronounced variation after $100 \mathrm{~nm}$ of deposited $\mathrm{SiO}_{2}$.

As for the TCF, Fig. 4 reveals that it follows the same tendency as the mass sensitivity. Both the longitudinal and shear TCFs start to deviate from their initial values with few $\mathrm{nm}$ of $\mathrm{SiO}_{2}$. This influence is more pronounced for the longitudinal than for the shear mode. Up to around $120 \mathrm{~nm}$ of deposited $\mathrm{SiO}_{2}$ we move around a relative constant deviation of $2 \mathrm{ppm} /{ }^{\circ} \mathrm{C}$ in both cases. This means variations of $2.8 \mathrm{kHz}$ and 5 $\mathrm{kHz}$ for the shear and longitudinal modes, respectively. Considering the values of our sensitivities $2.8 \mathrm{kHz}$ and $5 \mathrm{kHz}$ are already in the range of $\Delta \mathrm{m}=1 \mathrm{ng} / \mathrm{cm}^{2}$ detection, hence for accurate small weight detection the temperature of the device should be kept constant to avoid errors. On the contrary, if the target are bigger masses, then this error arising from TCFs variation could be negligible. 


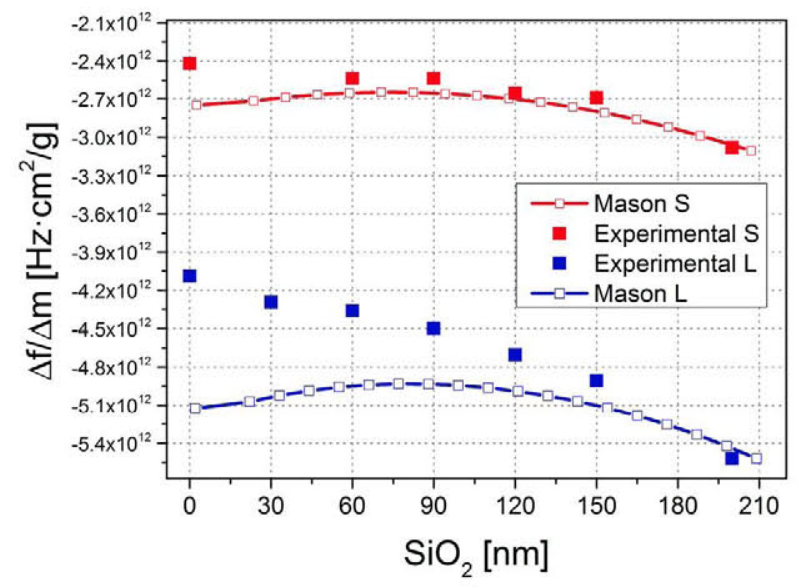

Fig. 3. Sensitivity evolution of the shear and longitudinal mode with deposited $\mathrm{SiO}_{2}$.

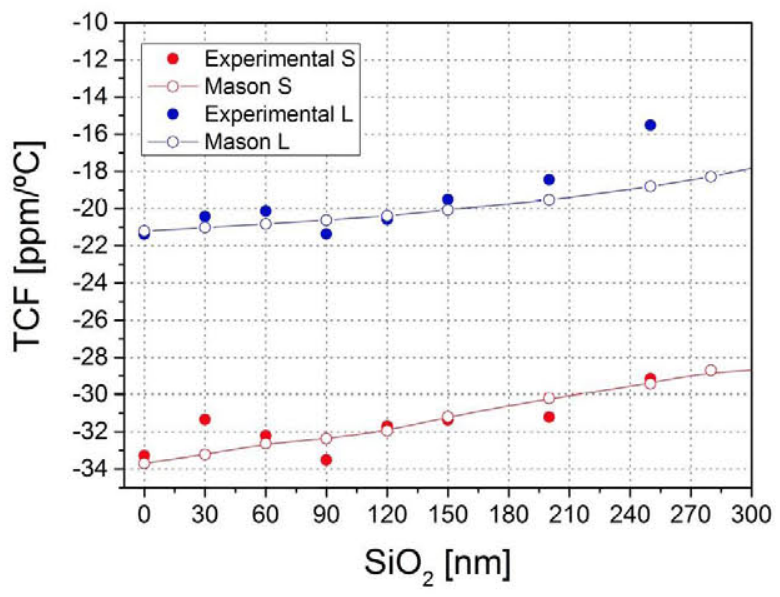

Fig. 4. TCF evolution of the shear and longitudinal mode with deposited $\mathrm{SiO}_{2}$.

\section{CONCLUSIONS}

The mass sensitivity of AlN-based SMRs varies with only few $\mathrm{nm}$ of deposited $\mathrm{SiO}_{2}$ on their active surface. This is in agreement with previous studies where they proved the mass sensitivity of FBARs is increased by adding low acoustic impedance materials as $\mathrm{SiO}_{2}$. Nevertheless, for a $\mathrm{SiO}_{2}$ deposition of up to approximately $100 \mathrm{~nm}$ the sensitivity can be considered constant even when the target are low masses in the $\mathrm{ng} / \mathrm{cm}^{2}$ range, since the error is less than the detection resolution. The TCF follows the same tendency, with a constant deviation of $2 \mathrm{ppm} /{ }^{\circ} \mathrm{C}$ up to around $150 \mathrm{~nm}$ of $\mathrm{SiO}_{2}$. In this case, this deviation is in the range of the sensitivity resolution, hence for very accurate sensing applications (i.e. detection of very light masses in the ng range) these effects should be taken into account. Depending on the characteristics of the deposited materials, these effects could vary. Hence they should be studied for each particular case. In all cases, if large masses or heavy species are to be detected, these effects can be disregarded, being the sensor available for reuse up to a certain limit.

\section{ACKNOWLEDGMENT}

This work was partially supported by Ministerio de Economía y Competitividad del Gobierno de España through project MAT2013-45957-R.

\section{REFERENCES}

[1] S. Rey-Mermet, R. Lanz, and P. Muralt, "Bulk acoustic wave resonator operating at $8 \mathrm{GHz}$ for gravimetric sensing of organic films," Sensors Actuators B Chem., vol. 114, no. 2, pp. 681-686, Apr. 2006.

[2] G. Sauerbrey, "Verwendung von Schwingquarzen zur Wagungdiinner Schichten und zur Mikrowagung," Zeitschrift fur Phys, vol. 155, pp. 206-222, 1959.

[3] G. Wingqvist, V. Yantchev, and I. Katardjiev, "Mass sensitivity of multilayer thin film resonant BAW sensors," Sensors Actuators A Phys., vol. 148 , no. 1 , pp. 88 $95,2008$.

[4] B. Ivira, P. Benech, R. Fillit, F. Ndagijimana, P. Ancey, and G. Parat, "Modeling for temperature compensation and temperature characterizations of BAW resonators at GHz frequencies," IEEE Trans. Ultrason. Ferroelectr. Freq. Control, vol. 55, no. 2, pp. 421-430, Feb. 2008.

[5] J. Bjurström, G. Wingqvist, V. Yantchev, and I. Katardjiev, "Temperature compensation of liquid FBAR sensors," $J$. Micromechanics Microengineering, vol. 17, no. 3, pp. 651-658, Mar. 2007.

[6] K. M. Lakin, K. T. McCarron, and J. F. McDonald, "Temperature compensated bulk acoustic thin film resonators," in 2000 IEEE Ultrasonics Symposium. Proceedings. An International Symposium (Cat. No.00CH37121), vol. 1, pp. 855-858. 